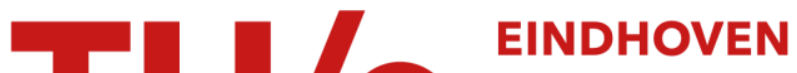 UNIVERSITY OF TECHNOLOGY
}

\section{Continuum methods for localised failure}

\section{Citation for published version (APA):}

Borst, de, R., Pamin, J., Schellekens, J. C. J., \& Sluys, L. J. (1995). Continuum methods for localised failure. In G. Baker, \& B. L. Karihaloo (Eds.), Fracture of brittle, disordered materials : concrete, rock and ceramics : proceedings of the International Union of Theoretical and Applied Mechanics (IUTAM) symposium, 20-24 September 1993, Brisbane, Australia (pp. 249-263). E\&FN SPON.

Document status and date:

Published: 01/01/1995

\section{Document Version:}

Publisher's PDF, also known as Version of Record (includes final page, issue and volume numbers)

\section{Please check the document version of this publication:}

- A submitted manuscript is the version of the article upon submission and before peer-review. There can be important differences between the submitted version and the official published version of record. People interested in the research are advised to contact the author for the final version of the publication, or visit the $\mathrm{DOI}$ to the publisher's website.

- The final author version and the galley proof are versions of the publication after peer review.

- The final published version features the final layout of the paper including the volume, issue and page numbers.

Link to publication

\section{General rights}

Copyright and moral rights for the publications made accessible in the public portal are retained by the authors and/or other copyright owners and it is a condition of accessing publications that users recognise and abide by the legal requirements associated with these rights.

- Users may download and print one copy of any publication from the public portal for the purpose of private study or research.

- You may not further distribute the material or use it for any profit-making activity or commercial gain

- You may freely distribute the URL identifying the publication in the public portal.

If the publication is distributed under the terms of Article 25fa of the Dutch Copyright Act, indicated by the "Taverne" license above, please follow below link for the End User Agreement:

www.tue.nl/taverne

Take down policy

If you believe that this document breaches copyright please contact us at:

openaccess@tue.nl

providing details and we will investigate your claim. 


\title{
16 CONTINUUM METHODS FOR LOCALISED FAILURE
}

\author{
R. de BORST \\ Delft University of Technology, Faculty of Civil \\ Engineering/Eindhoven University of Technology, Faculty of \\ Mechanical Engineering, The Netherlands \\ J. PAMIN, J.C.J. SCHELLEKENS and L.J. SLUYS \\ Delft University of Technology, Faculty of Civil Engineering, The \\ Netherlands
}

\begin{abstract}
Continuum approaches are reviewed which set out to model localised deformations that act as a precursor to final fracture in quasi-brittle materials. The role of material instabilities in triggering strain localisation is discussed as well as that of dispersive wave propagation in setting the strain profile in localisation bands (fracture process zones). Some numerical examples are given of structures made of concrete and fibre-reinforced polymers and ceramics in order to illustrate the discussion.
\end{abstract}

Keywords: Higher-order Continua, Softening, Fracture, Localisation, Finite Element Analysis

\section{Introduction}

The simulation of fracture processes in quasi-brittle materials is a complicated field of research. The inhomogeneous deformations that occur even in carefully executed servo-controlled experiments make the task of obtaining reliable and reproducible experimental results difficult and challenging at the same time for the experimentalist. However, the difficulties inherent in modelling the experimentally observed phenomena in a physically realistic manner and in a mathematically consistent fashion, and subsequently in translating this model into efficient and robust numerical simulation software also set a major task for theoreticians and numerical experts.

Essentially, two different approaches can be distinguished in modelling fracture processes in quasi-brittle materials like concrete, rock and ceramics. Firstly, we have discontinuum approaches, in which the crack is modelled by introducing geometric discontinuities in the numerical simulation. Secondly - and this will be the subject of this treatment - fracture and damage processes can be modelled by setting up appropriate constitutive models within the framework of continuum mechanics. This for instance enables modelling micro-cracks, voids and micro-structural defects at the continuum level.

A major problem when using a standard, rate-independent continuum for modelling degradation processes is that beyond a certain level of damage accumulation the governing set of partial differential equations changes type. In the static case the elliptic character of the set of partial differential equations is lost, while, on the other hand, in the dynamic case we observe a change of a hyperbolic set into an elliptic set. In both cases the rate boundary value problem becomes ill-posed and numerical solutions suffer from spurious mesh sensitivity.

The inadequacy of the standard, rate-independent continuum to model failure zones correctly is due to the fact that force-displacement relations measured in testing devices are simply mapped onto stress-strain curves by dividing the force and the elongation by the original loadcarrying area and the original length of the specimen respectively. This is done without taking into account the changes in the micro-structure that occur when the material is so heavily damaged as in fracture processes. Therefore, the mathematical description ceases to be a meaningful representation of the physical reality.

To solve this problem one must either introduce additional terms in the continuum 
description which reflect the changes in the micro-structure that occur during fracture, or one must take into account the viscosity of the material. In both cases the effect is that the governing equations do not change type during the damage evolution process and that physically meaningful solutions are obtained for the entire loading range. Another way to look upon the introduction of additional terms in the continuum description is that the Dirac distributions for the strain at failure are replaced by continuous strain distributions, which lend themselves for description by standard numerical schemes. Although the strain gradients are now finite, they may be very steep and the concentration of strain in a small area is then referred to as strain localisation or localisation of deformation.

This paper starts by reviewing the role of material instabilities in potentially destabilising structural behaviour and in causing loss of well-posedness of the rate-boundary value problem. Then, various methods that set out to cure the loss of well-posedness at damage evolution are elucidated, such as non-local approaches, gradient models, Cosserat models and the inclusion of rate-dependence. Finally the role of dispersion in setting the strain profile in localisation zones is reviewed.

\section{Material instabilities}

At the continuum level the driving forces behind localisation phenomena are material instabilities, that is, the constitutive relationship that relates stress to strain violates the stability criterion that the inner product of the stress rate $\dot{\sigma}_{i j}$ and the strain rate $\dot{\varepsilon}_{i j}$ is positive, Hill (1958), Maier and Hueckel (1979)!:

$$
\dot{\varepsilon}_{i j} \dot{\sigma}_{i j}>0 \text {. }
$$

Obviously, this inner product becomes negative when, in a uniaxial tension or compression test, the slope of the homogenised axial stress - axial strain curve is negative. We call this phenomenon strain softening. By using the terminology 'homogenised' we refer to the fact that initial flaws and boundary conditions necessarily induce a non-homogeneous stress state in a specimen. In particular during progressive failure of the specimen these flaws and local stress concentrations will cause strongly inhomogeneous deformations of the specimen. The procedure that is normally utilised to derive stress-strain relations, namely dividing the force by the virgin load-carrying area and dividing the displacement of the end of the specimen by the original length so as to obtain stress and strain respectively then no longer reflects what happens at a micro-level and loses physical significance. Nonetheless, we may still employ this procedure in a continuum description provided that we account for these micro-structural changes by including additional terms in the model.

There is also a class of material instabilities that can cause the inner product of stress rate and strain rate to become negative without the occurrence of strain softening as defined above. These instabilities can arise when the predominant load-carrying mechanism of the material is due to frictional effects such as in sands, rock joints and in pre-cracked concrete. At a phenomenological level such material behaviour usually results in constitutive models which, in a multiaxial context, have a non-symmetric relation between the stress-rate tensor and the strainrate tensor. This lack of symmetry is in itself sufficient to cause loss of material stability, even if the slope of the axial stress-strain curve is still rising, Rudnicki and Rice (1975).

Limiting the discussion to incrementally-linear stress-strain relations, that is the relation between stress rate $\dot{\sigma}_{i j}$ and strain rate $\dot{\varepsilon}_{k l}$ can be written as

$$
\dot{\sigma}_{i j}=D_{i j k l} \dot{\varepsilon}_{k l} \text {, }
$$

with $D_{i j k l}$ the material tangential stiffness tensor, inequality (1) can be formulated as

$$
\dot{\varepsilon}_{i j} D_{i j k l} \dot{\varepsilon}_{k l}>0 \text {. }
$$

The limiting case that the inequality of (3) is replaced by an equality, marks the onset of material instability. Mathematically, this is associated with loss of positive-definiteness of the 
material tangential stiffness tensor $D_{i j k l}$ :

$$
\operatorname{det}\left(D_{i j k l}+D_{k l i j}\right)=0 \text {. }
$$

Material instability can lead to structural instability. For a structure that occupies a volume $V$ Hill's definition (1958) guarantees stability if

$$
\int_{V} \dot{\varepsilon}_{i j} \dot{\sigma}_{i j} \mathrm{~d} V>0
$$

for all kinematically admissible $\dot{\varepsilon}_{i j}$. Obviously, violation of inequality (1), i.e. loss of maerial stability, may lead to violaion of identity (5), thus opening the possibility of structural instability. Accordingly, the mere existence of material instabilities (e.g. strain softening) may lead to structural instability, even in the absence of geometric destabilising terms.

In itself this observation does not explain the frequently reported pathological behaviour of finite element computations for constitutive models where positive-definiteness of $D_{i j k l}$ fails at some stage of the loading process. Indeed, the crucial consequence of the loss of positivedefiniteness of the material tangent operator $D_{i j k l}$ is not that it may cause structural instability, but that it may result in loss of ellipticity of the governing se of rate equations. Mathematically, loss of ellipticity occurs if

$$
\operatorname{det}\left(n_{j} D_{i j k l} n_{l}\right)=0 \text {, }
$$

where the summation convention with respect to repeated indices has been adopted. Physically, condition (6) indicates the existence of a discontinuity (with the normal vector $n_{i}$ ) in the velocity gradient and is coincident with Hill's condition for the propagation of plane acceleration waves in solids, Hill (1962).

Assuming small displacement gradients loss of material stability as expressed by (4) is a necessary condition for loss of ellipticity. To show this we first substitute the strain rate field that derives from a piecewise homogeneous deformation, cf. Knowles and Sternberg (1978), de Borst (1986):

$$
\dot{\varepsilon}_{i j}=1 / 2\left(n_{i} m_{j}+n_{j} m_{i}\right) \text {, }
$$

with $m_{i}$ an arbitrary vector, in the condition for loss of material stability, i.e. $\dot{\varepsilon}_{i j} D_{i j k l} \dot{\varepsilon}_{k l}=0$, so that $m_{i} n_{j} D_{i j k l} m_{k} n_{l}=0$. This identity holds for arbitrary $m_{i}$ if and only if

$$
\operatorname{det}\left(1 / 2 n_{j}\left(D_{i j k l}+D_{k l i j}\right) n_{l}\right)=0 \text {. }
$$

Because the real-valued eigenspectrum of $n_{j} D_{i j k l} n_{l}$ is bounded by the minimum and maximum eigenvalues of $1 / 2 n_{j}\left(D_{i j k l}+D_{k l i j}\right) n_{l}$, $\operatorname{det}\left(1 / 2 n_{j}\left(D_{i j k l}+D_{k l i j}\right) n_{l}\right)$ always vanishes prior to satisfaction of eq.(6). Since eq. (8) can only by satisfied if material stability is lost (eq.(4)), it follows that loss of ellipticity can only occur if we have loss of material stability.

Ellipticity is a necessary condition for well-posedness of the rate boundary value problem, in the sense that a finite number of linearly independent solutions are admitted, continuously depending on the data and not involving discontinuities, Benallal et al. (1991). It is emphasised that ellipticity is a local condition and is but one of the three conditions that are necessary for well-posedness of the rate boundary value problem. The other two conditions for wellposedness are satisfaction of the boundary complementing condition, which excludes the emergence of stationary surface waves (Rayleigh waves), and the satisfaction of the interfacial complementing condition, which excludes the emergence of stationary interfacial waves (Stonely waves), Needleman and Ortiz (1991).

\section{The standard continuum approach for localised failure computations}

The essential deficiency of the standard continuum model is most simply demonstrated by the example of a simple bar loaded in uniaxial tension, Figure 1 (Crisfield 1982, de Borst 1986). 


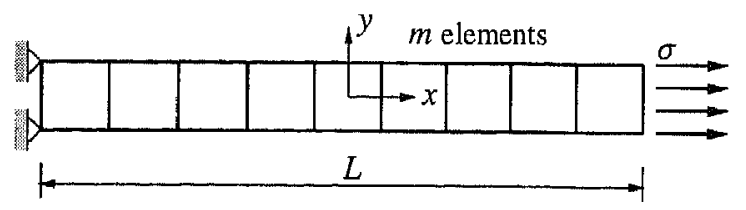

Figure 1. Strain-softening bar subject to uniaxial loading.
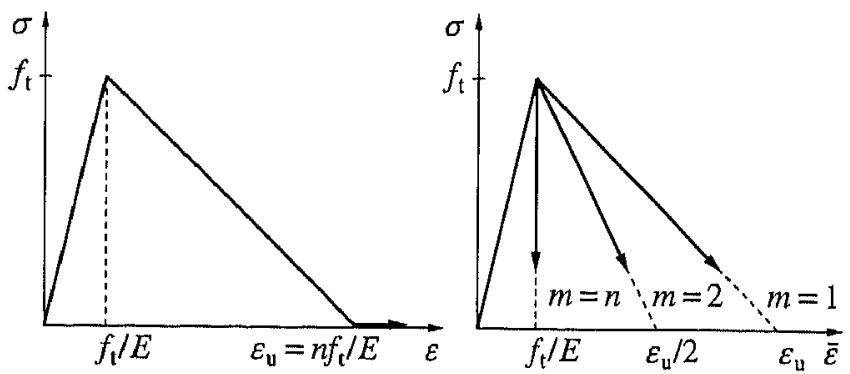

Figure 2. Stress-strain diagram (left) and response of an imperfect bar in terms of an stress-average strain curve (right).

Let the bar be divided into $m$ elements. Prior to reaching the tensile strength $f_{\mathrm{t}}$ a linear relation is assumed between the (normal) stress $\sigma$ and the (normal) strain $\varepsilon$ :

$$
\sigma=E \varepsilon,
$$

with $E$ Young's modulus. After reaching the peak strength a descending slope is defined in this diagram through an affine transformation from the measured load-displacement curve. The result is given in the left part of Figure 2, where $\varepsilon_{\mathfrak{u}}$ marks the point where the load-carrying capacity is totally exhausted. In the post-peak regime the constitutive model can thus be summarised as:

$$
\varepsilon=\varepsilon^{\mathrm{e}}+\varepsilon^{\mathrm{i}}
$$

which constitutes a decomposition of the strain into an elastic part $\varepsilon^{\mathrm{e}}$ :

$$
\varepsilon^{\mathrm{e}}=E^{-1} \sigma,
$$

and a contribution due to inelastic effects (eg, cracking or plastic slip)

$$
\varepsilon^{\mathrm{i}}=h^{-1}\left(\sigma-f_{\mathrm{t}}\right)
$$

where $h$ plays a role for the inelastic strain $\varepsilon^{\mathrm{i}}$ similar to that of $E$ for the elastic strains. In case of degrading materials $h<0$ and $h$ is termed a softening modulus. For simplicity sake $h$ is now assumed to be a constant (linear softening). Eq. (12) may also be thought of as an integrated form of the evolution equation for the stress rate after failure:

$$
\sigma=f_{\mathrm{t}}+h \varepsilon^{\mathrm{i}} \text {. }
$$

Now suppose that one element has a tensile strength that is marginally below that of the other $m-1$ elements. Upon reaching the tensile strength of this element failure will occur. In the other, neighbouring elements the tensile strength is not exceeded and they will unload elastically. The result on the average strain of the bar $\widetilde{\varepsilon}$ is plotted in the right part of Figure 2 for different discretisations of the bar. The results are fully dominated by the discretisation, and 

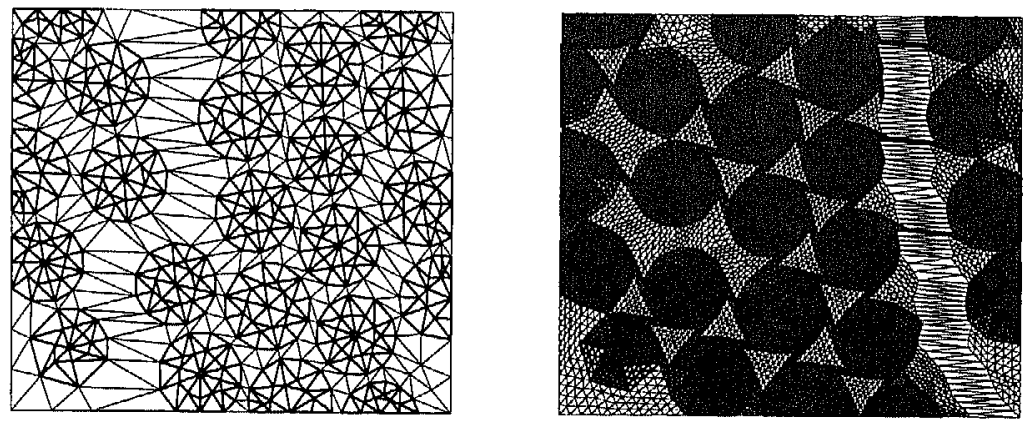

Figure 3. Deformed configurations for carbonfibre-reinforced ceramic. Left: coarse mesh ( 973 elements). Right: fine mesh (15568 elements).

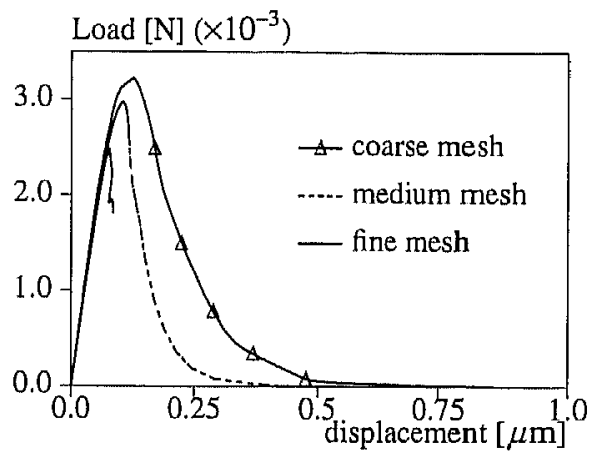

Figure 4. Load-displacement diagrams for carbonfibre-reinforced ceramic composite.

convergence to a 'true' post-peak failure curve does not seem to occur. In fact, it does occur, as the failure mechanism in a standard continuum is a line crack with zero thickness. The finite element solution of our continuum rate boundary value problem simply tries to capture this line crack, which results in localisation in one element, irrespective of the width of this element. The result on the load-average strain curve is obvious: for an infinite number of elements $(m \rightarrow \infty)$ the post-peak curve doubles back on the original loading curve.

The example of the micromechanical simulation of a carbonfibre-reinforced ceramic composite $(\mathrm{SiC})$ of Figure 3 (for details on the computation the reader is referred to Schellekens, 1992, and to Weihe, 1992, who prepared the finite element discretisations) is well suited to demonstrate that the above considerations for a simple one-dimensional example also hold for more complicated stress situations. Finite element simulations for three different degrees of refinement of the discretisation were run. As is shown in Figure 3 for the coarsest and the finest mesh the deformations always localise in a single row of elements and the load-deformation curve of Figure 4 shows an identical pattern as suggested in the load-displacement curve of Figure 2 for the one-dimensional bar.

From a physical point of view the above behaviour is unacceptable and when we adhere to continuum descriptions one must enrich the continuum by adding higher-order terms, either in space or in time, which can accommodate narrow zones of highly localised deformations. 


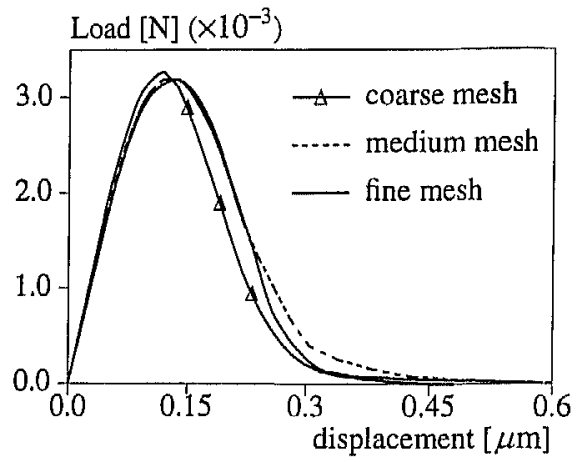

Figure 5. Load-displacement diagrams for carbonfibre-reinforced ceramic composite using a fracture-energy model.

\section{The fracture-energy 'trick'}

As an intermediate solution between using the standard continuum model and adding higherorder terms a number of authors (Pietruszczak and Mróz 1981, Bazant and Oh 1983, Willam 1984) have proposed to regard the area under the softening curve in the left part of Figure 2 as a material parameter, namely the fracture energy $G_{\mathrm{f}}$ :

$$
G_{\mathrm{f}}=\int \sigma \mathrm{d} u=\int \sigma \varepsilon(s) \mathrm{d} s
$$

Carrying out the integration for a linear softening diagram and assuming that the strains are constant over the band width (an assumption commonly made in numerical analyses) we arrive at the following relation between the strain $\varepsilon_{\mathrm{u}}$ at which the residual strength is exhausted, and the fracture energy $G_{f}$ :

$$
\varepsilon_{\mathrm{u}}=\frac{2 G_{\mathrm{f}}}{f_{\mathrm{t}} w},
$$

with $w$ the width of the localisation zone. The softening modulus $h$ is thus given by:

$$
h=\frac{w f_{\mathrm{t}}{ }^{2}}{2 G_{\mathrm{f}}},
$$

which is now a function of the element size. We now carry out an analysis for the tension bar of Figure 1 and give one element a tensile strength marginally below the other elements. Beyond the peak stress the tangential relation between the stress $\sigma$ in the bar and the displacement $u$
then reads:

$$
\frac{\dot{u} / L}{\dot{\sigma}}=\frac{1}{E}+\frac{1}{m h} .
$$

In a standard continuum localisation always occurs in one element, i.e. $w=L / m$. Using eq. (15) we can then rewrite eq. (16) as

$$
\frac{\dot{u} / L}{\dot{\sigma}}=\frac{1}{E}+\frac{2 G_{f}}{L f_{\mathrm{t}}^{2}},
$$

which shows that the solution in the post-peak regime is now only dependent upon the material parameters $E, G_{\mathrm{f}}$ and $f_{\mathrm{t}}$, and the structural size $L$.

The example of the carbonfibre-reinforced ceramic composite $(\mathrm{SiC})$ of Figure 3 also 


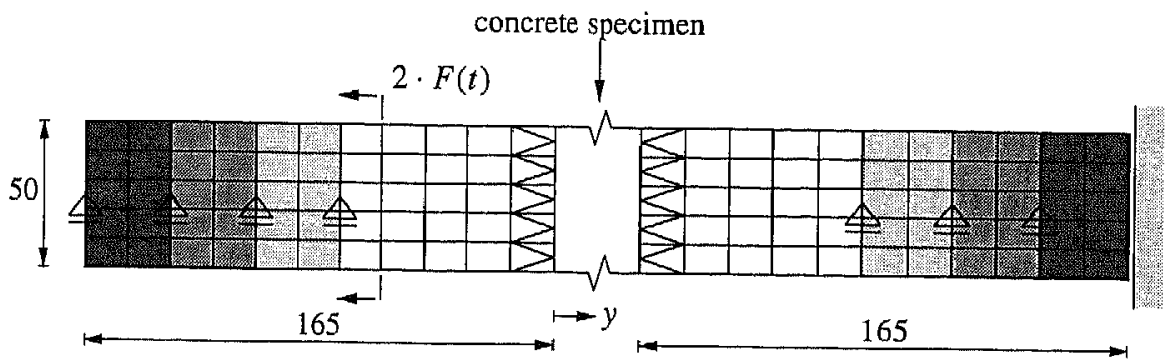

Figure 6. Numerical model of test set-up for FE analysis of Split-Hopkinson bar.
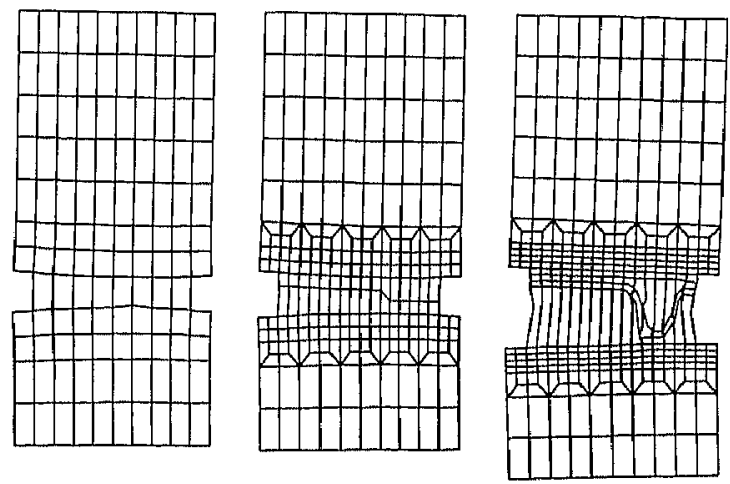

Figure 7. Displacement patterns of concrete specimen at $t=0.50 \cdot 10^{-3} \mathrm{~s}$.

serves as a nice illustration of the potential as well as the limitations of fracture energy type models. When we prescribe the fracture energy $G_{\mathrm{f}}$ as an additional material parameter Figure 5 shows that the global load-displacement response now indeed becomes insensitive to the discretisation. However, locally nothing has altered and localisation still takes place in one row of elements. This is logical, since the loss of ellipticity occurs at a local level, even though the energy that is dissipated remains constant by adapting the softening modulus to the element size. For numerical simulations this implies for instance that severe convergence problems are usually encountered if the mesh is refined or if in addition to matrix failure the possibility of interface debonding between matrix and fibres is modelled by inserting interface elements in the numerical model. Also, the frequently reported observation still holds that the localisation zones are biased by the discretisation and tend to propagative along the mesh lines. This can be nicely demonstrated with the example of impact loading a concrete specimen in a Split-Hopkinson device, Figure 6 (Sluys 1992). The results for the deformed specimen at failure is shown in Figure 7 for three different discretisations in the region between the notches. We observe a clear spurious localisation pattern with the localisation concentrated in a single band of elements which generally follows the mesh lines and occasionally jumps from one row to the next and back without any physical motivation.

\section{Non-standard continuum models}

The deficiency of the standard continuum model with regard to properly describing strain localisation can be overcome by introducing higher-order terms in the continuum description, which 
are thought to reflect the microstructural changes that take place at a level below the continuum level. Examples of such changes are void formation in metals and crack bridging phenomena, e.g., Van Mier (1991) in the context of concretes. Essentially, one then departs from the concept of a 'simple' solid (Noll, 1958) which has been the starting point for virtually all modern devel-
opments in continuum mechanics.

A number of suggestions have been put forward for non-standard continuum descriptions that are capable of properly incorporating failure zones. These include the non-local damage model of Pijaudier-Cabot and Bazant (1987), the use of the Cosserat model (Mühlhaus and Vardoulakis 1987, de Borst 1991, 1993) and the gradient models (Aifantis 1984, Schreyer and Chen 1986, Lasry and Belytschko 1988, Mühlhaus and Aifantis 1991, de Borst and Mühlhaus 1992). Here we will limit ourselves to a brief discussion and an example of a gradient-enhanced Rankine plasticity model (Pamin 1993). The essential feature of gradient plasticity theory is that the yield function not only depends upon the stress $\sigma$ and an equivalent inelastic strain measure $\gamma^{i}$,
but that there is also a dependence upon gradients of $\gamma^{i}$, e.g., the Laplacian:

$$
f=f\left(\sigma, \gamma^{\mathrm{i}}, \nabla^{2} \gamma^{\mathrm{i}}\right) \text {. }
$$

If we denote by $\sigma_{1}$ the major principal stress and by $\bar{\sigma}$ the instantaneous tensile strength, then

$$
f=\sigma_{1}-\tilde{\sigma}\left(\gamma^{\mathrm{i}}, \nabla^{2} \gamma^{\mathrm{i}}\right) \text {, }
$$
When it is further assumed that the dependence upon the gradient term is linear - the simplest
possible case - then eq. (19) further reduces to

$$
f=\sigma_{1}-\bar{\sigma}\left(\gamma^{\mathrm{i}}\right)-\bar{c} \nabla^{2} \gamma^{\mathrm{i}}
$$

In the example calculations that will be presented below $\bar{c}$ has been taken proportional to the rate of hardening (or in the present case softening): $\bar{c}=l^{2} \partial \bar{\sigma} / \partial \gamma^{\mathrm{i}}$. The material parameter $l$ has the dimension of length and represents the gradient influence. For $l=0$ the standard plasticity
model is recovered.

The gradient-dependent Rankine plasticity model has been applied to four-point bending tests on plain concrete notched beams. The experimental results are from Hordijk (1991), who has also proposed the nonlinear softening function that has been used and has provided the material data: Young's modulus $E=40 \mathrm{GPa}$ and $f_{\mathrm{t}}=3.3 \mathrm{MPa}$. The Poisson's ratio $v$ has been assumed to be equal to zero, and the internal length scale and equivalent fracture strain at complete strength degradation have been taken as $l=3 \mathrm{~mm}$ and $\varepsilon_{\mathrm{u}}=9.92 \cdot 10^{-3}$, respectively. The loading configuration including some aspects of the numerical discretisation are shown in Figthe employed mixed finite element formulation are given by points have been used. Details of Pamin (1993).

The results of the finite element analyses are presented in Figures 9 and 10 . Figure 9 which gives the overall load-displacement responses of the beams, shows that there is a good
correspondence between numerical simulation notch depth of $30 \mathrm{~mm}$. Moreover, the results are and experiment, especially for the case of a cretisation. The simulation with 155 elements are completely insensitive with respect to the dis475 elements. This observation can also be yields exactly the same results as the case with equal equivalent fracture strain at the final be drawn from Figure 10, which gives contours of paritive studies were undertaken with a $90 \%$ reduced load level. It is finally noted that these comby Pamin (1993) this modelling trick just marginally affects the rensile strength $f_{t}$. As shown

\section{Rate-dependent continuum models}

From a physical point of view the introduction of rate dependence is perhaps the most natural way to regularise ill-posed initial value problems which arise because of the introduction of damage or frictional effects. Here we adopt a simple, linear rate-dependent smeared crack 


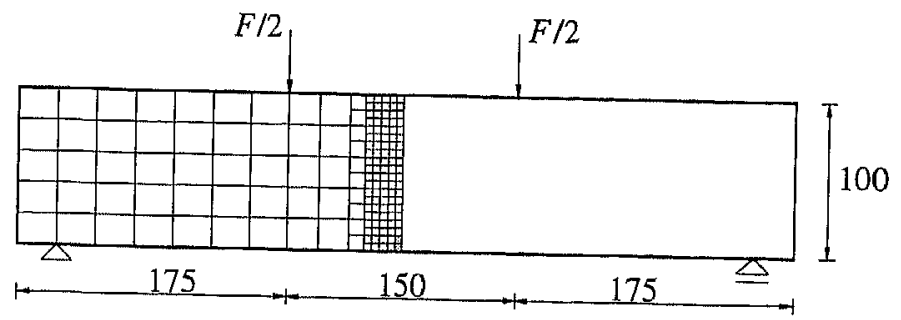

Figure 8. Four-point bending test on a plain concrete beam (Hordijk, 1991).

Force $[\mathrm{kN}]$

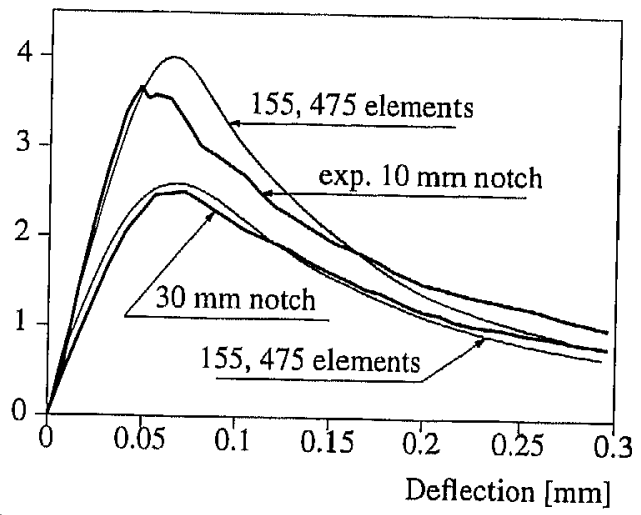

Figure 9. Computed and experimentally obtained load-displacement diagrams.
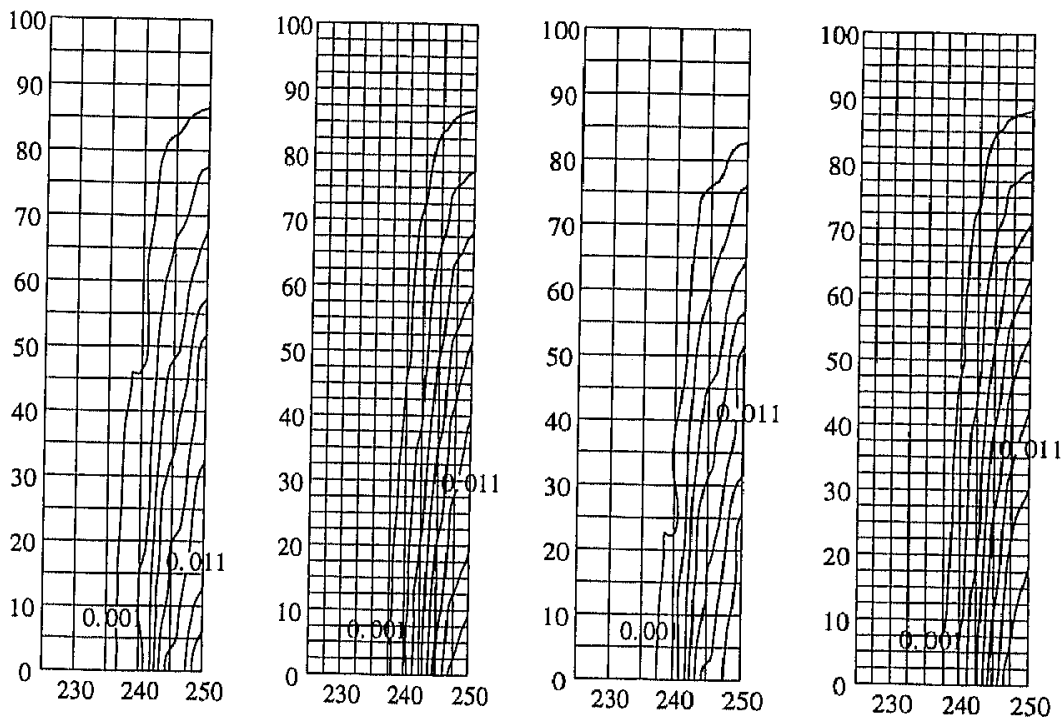

Figure 10. Contour plots of equivalent fracture strain for the final states (10 $\mathrm{mm}$ notch on the left, $30 \mathrm{~mm}$ notch on the right). 

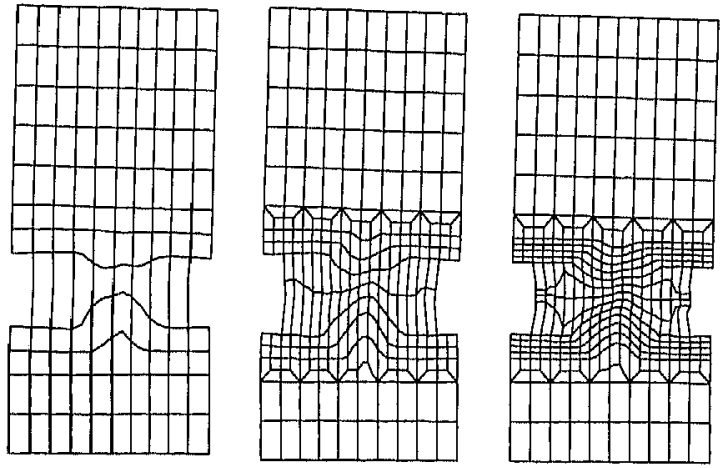

Figure 1I. Displacement patterns for rate-dependent analysis at $t=0.45 \cdot 10^{-3} \mathrm{~s}$.

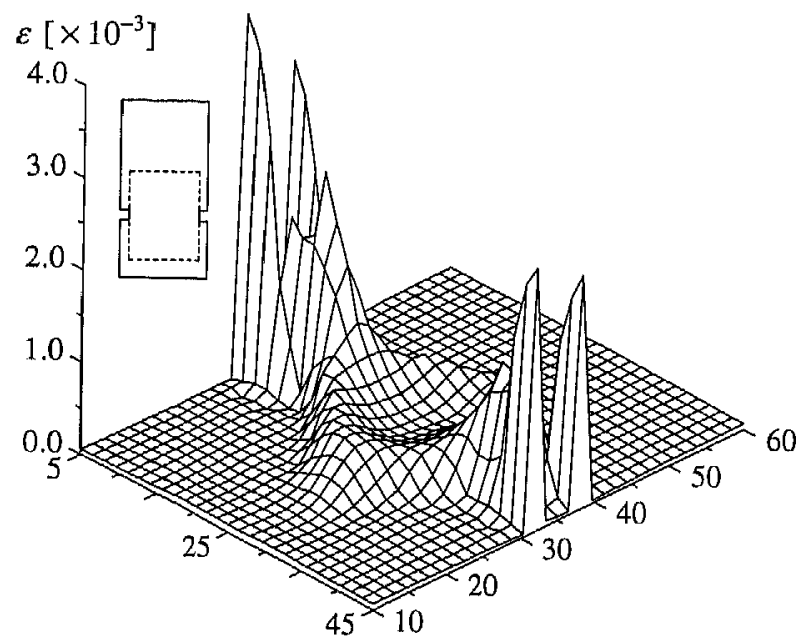

Figure 12. Axial strain profile in the notched area at $t=0.45 \cdot 10^{-3} \mathrm{~s}$.

model as developed by Sluys (1992). In it the major principal stress degrades according to

$$
\sigma=f_{\mathrm{t}}+h \varepsilon^{\mathrm{i}}+m \frac{\partial \varepsilon^{\mathrm{i}}}{\partial t}
$$

with $m$ a rate-sensitivity parameter which has been taken equal to $0.1 \mathrm{Ns} / \mathrm{mm}^{2}$ in the calculations presented below. As in the gradient-dependent model (cf. eq. (20)) the present model can be considered as a perturbation on the evolution equation (13).

Using the rate-dependent smeared crack model as defined in eq. (21) the experiment of a concrete specimen under impact loading in a Split-Hopkinson bar (cf. Figures 6 and 7) has been reanalysed. The incremental displacement patterns are shown in Figure 11. The most striking difference with the displacement pattern of Figure 6 is that localisation now does not proceed along the element lines and is no longer confined to the rows of elements between the notches. This is even more obvous when the strains in the vertical direction are plotted $\left(\varepsilon_{y y}\right)$ as has been 
done in Figure 12. We observe a clear branching of the cracks.

A basic drawback of the use of viscosity to restore well-posedness of the rate boundary value problem is the fact that the regularising influence gradually vanishes for the rateindependent limit or for very slow processes. This is shown below for the case of matrix failure in a carbon-epoxy composite. It has been assumed that a Duvaut-Lions softening viscoplasticity model with a Von Mises flow contour could be utilised to model the matrix failure.

In the composite a periodic arrangement of the fibres is assumed which results in a natural choice for the reference volume element (RVE). The unidirectional composite (50\% fibre volume fraction) is loaded in transverse tension as shown in Figure 13. The material properties of the Apollo IM 43-750 carbon fibres and the Ciba-Geigy Araldite Epoxy matrix are given by Schellekens and de Borst (1993).

By virtue of symmetry only a quarter of the RVE had to be discretised using 170, 336 and 1348 quadratic plane-strain triangular elements respectively. In the vertical direction free contraction of the RVE is allowed whereas an equal displacement constraint was imposed on the nodes of the right boundary. The response of the three meshes was calculated for different values of the loading rate $\dot{\varepsilon}_{11}$. The results of this mesh-refinement study are presented in Figure 14 in which the load-displacement curves of the right boundary of the RVE is shown. They confirm that for low loading rates some spurious discretisation effects again enter. Interestingly enough, this effect is less significant when interface elements are included in the model to simulate debonding between matrix and fibres. Apparently, the failure mechanism then changes such that the inherent mesh dependence for slow loadings becomes less important. These results for the viscoplastic continuum are in sharp contrast with those of the fracture-energy based continuum model, where no converged results could be obtained for the case that a softening model for the matrix failure was combined with an interface model for debonding.
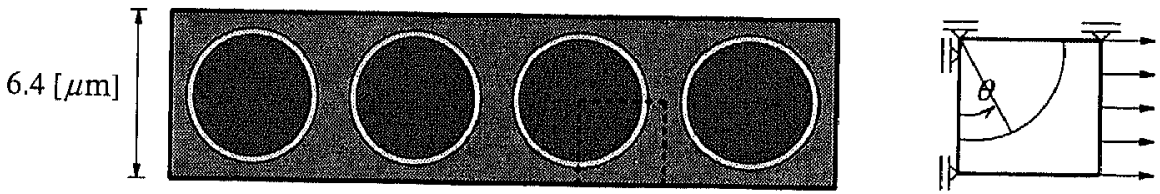

Figure 13. Geometry of RVE of a carbon-epoxy unidirectional composite.
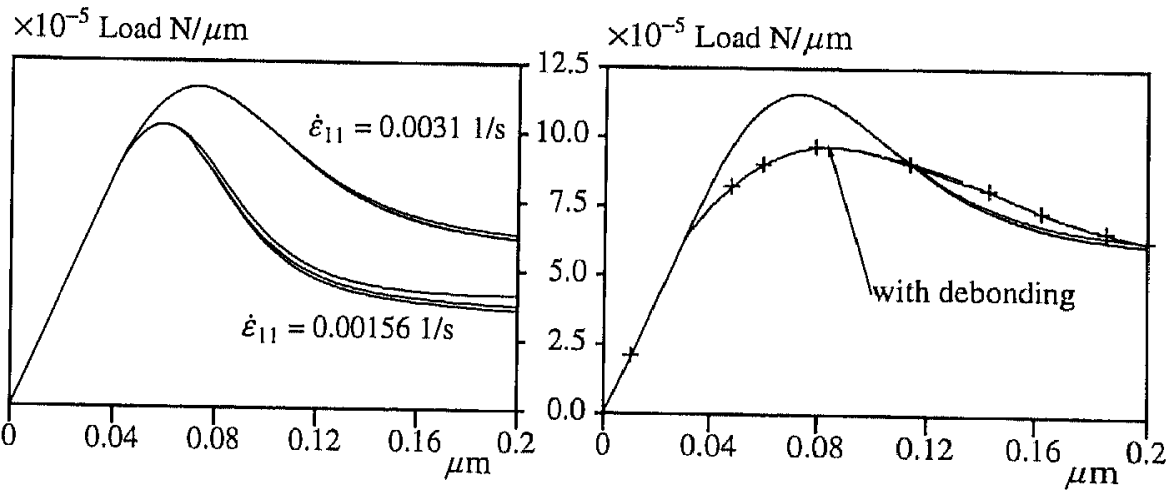

Figure 14. Load-displacement curves for the right boundary of the RVE.

Left: Effects of strain rate for the case without debonding.

Right: Effect of the inclusion of debonding on the structural response, 

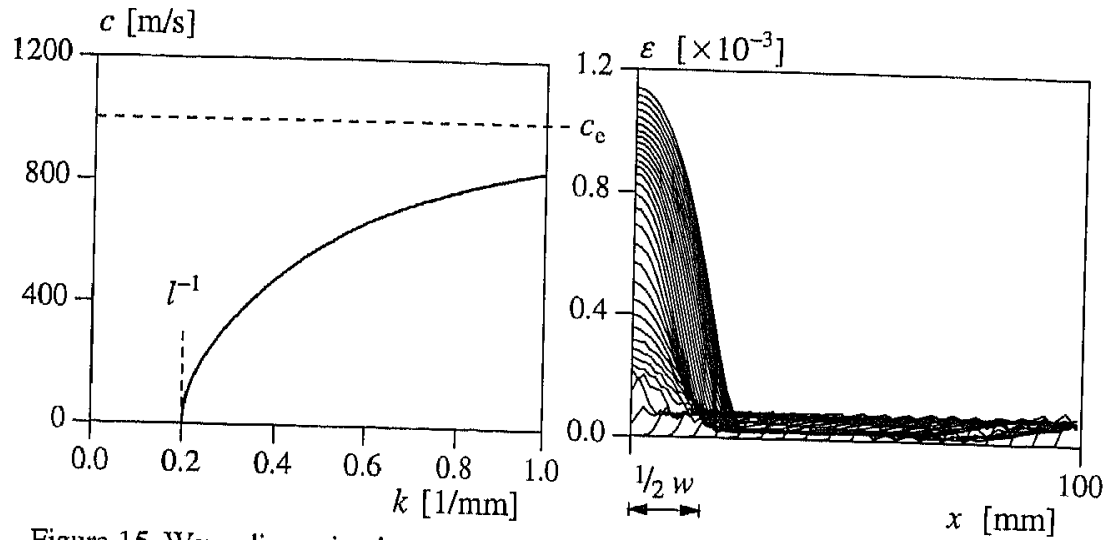

Figure 15. Wave dispersion in a one-dimensional bar with gradient plasticity.

Left: Phase velocity as a function of the wave number.

Right: Stroboscopic picture of development of the strain profile.

\section{Wave dispersion and localisation}

All higher-order continua as well as rate-dependent continuum models share the property that, unlike the standard, rate-independent continuum, wave propagation becomes dispersive, that is, waves with different wave numbers propagate with different velocities and, consequently, the shape of a pulse is altered during propagation. This phenomenon, which is of crucial importance for modelling localisation under dynamic loading conditions, will be discussed below for the case of a one-dimensional gradient-dependent elastoplastic bar.

For a one-dimensional continuum and assuming linear softening the gradient plasticity theory of eq. (20) reduces to

$$
\sigma=f_{\mathrm{t}}+h \varepsilon^{\mathrm{i}}+\bar{c} \frac{\partial^{2} \varepsilon^{\mathrm{i}}}{\partial x^{2}}
$$

To analyse wave propagation in a gradient-dependent one-dimensional element we differentiate eq. (22) with respect to $t$ and combine the result with the constitutive equations (10), (11), the $\partial \dot{\sigma} / \partial x=\rho \partial^{2} v / \partial t^{2}$ with $\rho$ the mass densitye axial velocity, and the equation of motion we obtain

$$
-\bar{c}\left(\frac{\partial^{4} v}{\partial x^{4}}-\frac{1}{c_{\mathrm{e}}^{2}} \frac{\partial^{4} v}{\partial x^{2} \partial t^{2}}\right)+\frac{E+h}{c_{\mathrm{e}}^{2}} \frac{\partial^{2} v}{\partial t^{2}}-h \frac{\partial^{2} v}{\partial x^{2}}=0,
$$

with $c_{\mathrm{e}}=\sqrt{E / \rho}$ the bar wave velocity. Substitution of the general form of a single harmonic
wave

$$
\nu(x, t)=A \exp (\mathrm{i}(k x-\omega t))
$$

with $\omega$ the angular frequency and $k$ the wave number, into the wave equation (23) gives the dispersion relation for the gradient-dependent bar

$$
\bar{c} k^{4}-\bar{c} / c_{\mathrm{e}}^{2} k^{2} \omega^{2}+(E+h) / c_{\mathrm{e}}^{2} \omega^{2}-h k^{2}=0 .
$$

Considering the positive root for $\omega$ 


$$
\omega=c_{\mathrm{e}} \sqrt{\frac{h-\bar{c} k^{2}}{E+h-\bar{c} k^{2}}} k,
$$

it becomes clear that the classical non-dispersive relation (cf. Sluys, 1992) is recovered when $\bar{c} \rightarrow 0$. The phase velocity $c=\omega / k$ of the harmonic wave reads

$$
c=c_{\mathrm{e}} \sqrt{\frac{h-\bar{c} k^{2}}{E+h-\bar{c} k^{2}}} .
$$

which relation has been plotted in the left part of Figure 15. The phase speed $c$ depends on the wave number $k$ and, consequently, wave propagation is dispersive for the gradient-dependent bar. Owing to the fact that different harmonic waves propagate with different velocities the shape of a pulse is altered and, in contrast to the standard continuum model, a loading wave can be transformed into a stationary localisation wave. For a gradient-dependent model the phase speed therefore not necessarily becomes imaginary at the onset of softening as in a standard continuum model. From eq. (27) it follows that the phase velocity remains real if

$$
k \geq \sqrt{\frac{h}{\bar{c}}} \text { and thus } \lambda \leq 2 \pi l \text {, with } l=\sqrt{\frac{\vec{c}}{h}} .
$$

The parameter $l$ is the internal length scale in the gradient-dependent model. If $k<l^{-1}$ or wave length $\lambda>2 \pi l$ we recover the situation in which a disturbance $\delta v$ is unbounded and stability in the sense of Lyapunov is lost (i.e. a small disturbance of boundary data results in large changes of the response). However, strain-softening regions remain small and no wave lengths larger than $2 \pi l$ can occur because they do not fit within the strain-softening region. Consequently, all phase velocities remain real because the first-order wave with the lowest wave number (largest wave length) has a wave number which is larger than the critical value in eq. (28). In a numerical simulation we observe that all higher frequencies which are present in a loading wave vanish under the influence of nonlinear material behaviour and we obtain a stationary harmonic localisation wave with a width equal to the maximum wave length $w=\lambda=2 \pi l$. This is shown clearly in the right part of Figure 15, which gives a stroboscopic picture of the strain profiles along the bar. Upon reflection of the wave to the left (fixed) boundary of the bar the yield limit is exceeded and softening occurs. As observed from the right part of Figure 15 initially a sine-like strain profile develops with a width equal to $w=2 \pi l$, which is in agreement with the results of the dispersion analysis. A new stage in the localisation process is entered when the strength contribution due to local softening has vanished in some part of the localisation zone. According to eqs. (27) and (28) the wave length $\lambda$ then increases and the wave speed becomes positive. The localisation zone is no longer stationary, but extends.

\section{Concluding remarks and outstanding problems}

In this contribution current developments have been reviewed for modelling localisation and fracture via continuum methods. Various promising approaches exist, but there is no such a thing like a panacea which cures the shortcomings of standard, rate-independent continua upon the introduction of strain softening and/or non-symmetry in the constitutive rate equations.

No completeness is claimed. This holds true especially with respect to outstanding problems which hamper the effective and accurate numerical prediction of the failure and postfailure behaviour of structures. In the authors' opinion the most pressing issues that require further attention for failure computations are:

- The proper determination of the additional model parameters that emerge in the higherorder and rate-dependent continuum models when compared to the classical approach. Especially in higher-order continua this problem is not solved easily, since the additional parameters are not directly derivable from elementary tests such as uniaxial or triaxial 
tension or compression tests. Even if one would be able to carry out a test on a perfect procimen, so that homogeneous deformations would occur throughout the entire loading programme, these parameters could not be measured because for homogeneous deformaceed in a semi-inverse manner, whereby ther continuum models. Therefore, one must proare fitted in the post-peak regime.

- The steep (but finite!) strain gradients that occur in higher-order and rate-dependent continua during failure require that very fine meshes are used to capture the failure mode ers, then the use of adaptive mesh refried out on nowadays' or even tomorrow's computconditio sine qua non. A problem is thent techniques or spectral overlay methods is a ment in inelastic, non-standard continu. Althelopment of proper criteria for mesh refinean easy task.

- The combination deformations. Little work has been dods with higher-order continuum models for localised tions (Carmeliet 1992).

\section{References} Aifantis, E.C. (1984) On the microstructural original of certain inelastic models. J. Eng. Mater
Technol., 106, 326-334.

Bazant, Z.P. and Oh, B. (1983) and Structures, 16, 155-177.

Benallal, A, Billardon, $\mathrm{R}$ and and interfaces of solids. als: Theory and Applications, (Ed. Desai, Conf. Constitutive Laws for Engineering Materi-

de Borst, R. (1986) Non-Linear And. Desai, C.S.), pp. 387-390, Tucson, Arizona. of Technology, Delft. de Borst, R. (1991) Simu Engineering Computations, 8, 317-332

de Borst, R. and Mijh rithmic aspects. Int. I Num . (1992) Gradient-dependent plasticity: formulation and algo-

de Borst, R. (1993) A generalistion. Eng., 35, 521-539. Mech. Eng., 103, 99-122.

Carmeliet J (1992) Dum tation, Catholic University of Leuven, Leefselgewapende pleisters voor buitenisolatie. Disser-

Crisfield, M.A. (1982) Local instabilities in then and slabs. Proc. Instn. Civ. Engrs, Part the non-linear analysis of reinforced concrete beams Hill, R. (1958) A general theory of, Part 2, 73, 135-145.

Phys. Solids, 6, 236-249.

Hill, R. (1962)

Hordijk, D.A. (1991) Local waves in solids. J. Mech. Phys. Solids, 10, 1-16. Technology, Delft.

of concrete. Dissertation, Delft University of contimuous deformationg. E. (1978) On the failure of ellipticity and the emergence of dis-

Lasry, D. and Belytschko, T. (1988) Localization elastostatics. J. Elasticity, 8, 329-379. Structures, 24, 581-597.

Maier, G. and Hueckel, T. (1979) Nonassociated and coupled rock-like materials. Int. J. Rock Mech.ciated and coupled flow rules of elastoplasticity for

Mier, J.G.M. van (1991) Mode-I fech. Min. Sci. \& Geomech. Abstr., 16, 77-92. interface grain bridging. Cement and 
Mühlhaus, H.-B. and Vardoulakis, I. (1987) The thickness of shear bands in granular materials. Geotechnique, 37, 271-283.

Mühlhaus, H.-B. and Aifantis, E.C. (1991) A variational principle for gradient plasticity. Int. J. Solids Structures, 28, 845-858.

Needleman, A. and Ortiz, M. (1991) Effect of boundaries and interfaces on shear-band localization. Int. J. Solids Structures, 28, 859-878.

Noll, W. (1958) A mathematical theory of the mechanical behaviour of continuous media. Arch. Rat. Meth. Anal., 2, 197-226.

Pamin, J. (1993) Mesh sensitivity in gradient dependent softening plasticity: II. Application of Rankine and Drucker-Prager yield criteria. Report 25-2-93-2-14, Delft University of Technology, Delft.

Pietruszczak, S. and Mróz, Z. (1981) Finite element analysis of deformation of strain softening materials. Int. J. Num. Meth. Eng., 17, 327-334.

Pijaudier-Cabot, G. and Bazant, Z.P. (1987) Nonlocal damage theory. ASCE J. Eng. Mech., 113, 1512-1533.

Rudnicki, J.W. and Rice, J.R. (1975) Conditions for the localization of deformation in pressuresensitive dilatant solids. J. Mech. Phys. Solids, 23, 371-394.

Schellekens, J.C.J. (1992) Computational Strategies for Composite Structures. Dissertation, Delft University of Technology, Delft.

Schellekens, J.C.J. and de Borst, R. (1993) The application of interface elements and enriched or rate-dependent continua to micro-mechanical analyses of fracture in composites. Computational Mechanics, in press.

Schreyer, H.L. and Chen, Z. (1986) One-dimensional softening with localization, J. Appl. Mech. 53, 791-979.

Sluys, L.J. (1992) Wave Propagation, Localisation and Dispersion in Softening Solids. Dissertation, Delft University of Technology, Delft.

Weihe, S. (1992) NUmerical simulation of composite failure. Part I: Random generation of $2 D$ fibre composites. ISD Report 92-W-01, University of Stuttgart, Stuttgart.

Willam, K.J. (1984) Experimental and computational aspects of concrete fracture, in Proc. Int. Conf. Computer Aided Analysis and Design of Concrete Structures, (eds F. Damjanić et al.), Pineridge Press, Swansea, Part 1, pp. 33-70. 